\title{
Reactive oxygen species spermine metabolites generated from amine oxidases and radiation represent a therapeutic gain in cancer treatments
}

\author{
ROBERTO AMENDOLA ${ }^{1}$, MANUELA CERVELLI ${ }^{2}$, EMILIANO FRATINI ${ }^{1}$, DAVIDE E. SALLUSTIO ${ }^{1}$, \\ GIAMPIERO TEMPERA $^{3}$, TAICHI UESHIMA ${ }^{3}$, PAOLO MARIOTTINI ${ }^{2}$ and ENZO AGOSTINELLI ${ }^{3}$
}

\author{
${ }^{1}$ ENEA, CR Casaccia, UT Bio-Rad RAB, I-00123 Rome; ${ }^{2}$ Department of Biology, University of 'Roma Tre', I-00146 Rome; \\ ${ }^{3}$ Institute Pasteur Cenci-Bolognetti Foundation and Department of Biochemical Sciences 'A. Rossi Fanelli', \\ University of Rome 'Sapienza' and CNR, Biology and Molecular Pathology Institutes, I-00185 Rome, Italy
}

Received April 24, 2013; Accepted June 7, 2013

DOI: $10.3892 /$ ijo.2013.2013

\begin{abstract}
The most frequent interventions in cancer therapy are currently the destruction of cells by irradiation or administration of drugs both able to induce radical formation and toxic metabolites by enzyme-catalyzed reactions. The aim of this study was to determine the cell viability of cells undergoing a DNA damage threshold accomplished by ROS overproduction via both ectopic expression of murine spermine oxidase (mSMOX) and bovine serum amine oxidase (BSAO) enzymes. Low dose of $\mathrm{X}$-irradiation delivers a challenging dose of damage as evaluated in proficient Chinese hamster AA8 cell line and both deficient transcription-coupled nucleotide excision repair (NER) UV61 cells and deficient base excision repair (BER) EM9 cells, at 6 and $24 \mathrm{~h}$ after exposure. The priming dose of ROS overexposure by $\mathrm{mSMOX}$ provokes an
\end{abstract}

Correspondence to: Dr Roberto Amendola, ENEA, CR Casaccia, UT Bio-Rad RAB, Via Anguillarese 301, I-00123 Rome, Italy

E-mail: roberto.amendola@enea.it

Abbreviations: ALDH, aldehyde dehydrogenase; BER, baseexcision-repair; BSA, bovine serum albumin; BSAO, bovine serum amine oxidase; DOX, doxorubicin; DX, doxorubicin-resistant; FBS, fetal bovine serum; FCM, flow cytometry; HEPES-BSS, HEPES-buffered balanced salt solution; $\mathrm{H}_{2} \mathrm{O}_{2}$, hydrogen peroxide; $\mathrm{H}_{2}$ DCFDA, 2',7'-dichlorodihydroflurescein; IU, international units; LNT, linear no-threshold; MDR, multidrug-resistant; mSMOX, mouse spermine oxidase; $\mathrm{NAD}^{+}$, nicotine adenine dinucleotide; NER, nucleotide-excision-repair; PA, polyamine; PBS, phosphatebuffered saline; P-gp, P-glycoprotein; ROS, reactive oxygen species; SD, standard deviation; SDS/PAGE, sodium dodecyl sulphate/ polyacrylamide gel electrophoresis; SPM, spermine; TEM, transmission electron microscopy; TUNEL, 3'-terminal deoxytransferase; WT, wild-type

Key words: spermine, reactive oxygen species, neuroblastoma, base-excision-repair, nucleotide-excision-repair, bovine serum amine oxidase, multidrug-resistant, cytotoxicity adaptive response in $\mathrm{N} 18 \mathrm{TG} 2$, AA8 and EM9 cell lines at $24 \mathrm{~h}$. Interestingly, in the UV61 cells, ROS overexposure by mSMOX delivers an earlier adaptive response to radiation. The enzymatic formation of toxic metabolites has mainly been investigated on wild-type (WT) and multidrug-resistant (MDR) cancer cell lines, using and spermine as substrate of the BSAO enzyme. MDR cells are more sensitive to the toxic polyamine metabolites than WT cells, thus indicating a new therapeutic strategy to overcome MDR tumors. Since SMOX in mammals is differentially activated in a tissue-specific manner and cancer cells can differ in terms of DNA repair and MDR capabilities, it could be of interest to simultaneously treat with very low dose of X-rays and/or to alter SMOX metabolism to generate a differential response in healthy and cancer tissues.

\section{Introduction}

Mammalian cells evolved and constantly live in a highly reactive oxidative environment. In the mammalian organism $\mathrm{H}_{2} \mathrm{O}_{2}$ has a central position within the reactive oxygen species (ROS) family. Its formation by several reactions and its controlled inactivation is the basis of redox homeostasis $(1,2)$, since free radicals are highly cytotoxic. Oxidative stress is the result of interactions with macromolecules among highly reactive hydrogen peroxide $\left(\mathrm{H}_{2} \mathrm{O}_{2}\right)$ and singlet oxygen $\left({ }^{1} \mathrm{O}_{2}\right)$, the radicals superoxide anion $\left(\mathrm{O}_{2}^{-}\right)$and the hydroxyl radical (HO). Besides the major interest in identification and advancement of compounds which are either radical scavengers or antioxidants, ROS can alternate between a positive and negative cellular outcomes (3). Insufficient ROS defense mechanisms are mutagenic and promote cell death, apoptosis and autophagy (4). Recently autophagy and apoptosis stimulus by the presence of an $\mathrm{H}_{2} \mathrm{O}_{2}$-induced pathway in human primary and tumor cell lines and in primary cells (5), was demonstrated. Indeed, ROS may cause unfavorable cellular outcome or a positive one to eliminate DNA damaged cells and thus preventing carcinogenesis. From a mechanistic point of view, a clear and elegant example of the ROS pivotal effect on cellular outcome has been described by tumor necrosis factor (TNF- $\alpha$ ) binding to 
tumor necrosis factor receptor 1 (TNFR1). This binding can induce cell proliferation and cell death, both mediated, by Jun $\mathrm{N}$-terminal kinase (JNK) activation which is enhanced by ROS production (6). The natural polyamines (PA), putrescine (PUT), spermidine (SPD) and spermine (SPM) are ubiquitous low-molecular weight aliphatic cations essential for eukaryotic cells $(7,8)$ and tumor cell PA depletion was associated with the downregulation of $\mathrm{Bcl}-2$ protein and an increase of reactive oxygen species $(9,10)$. To adequately preserve concentrations, PA metabolism is strictly regulated by the interconversion stepwise degradation, which is responsible for the oxidant by-products $(8,11)$. Increasing interest has been posed on the SPM amino oxidase (SMOX) and BSAO enzyme activities, since SPM catabolic degradation has been found closely related to DNA oxidation and apoptosis, mainly via hydrogen peroxide $\left(\mathrm{H}_{2} \mathrm{O}_{2}\right)$ production (12-15). High SMOX activity provokes low level of SPM and the inhibition of the interactions with DNA, thus causing sensitivity to ionizing radiation exposure and cell death $(15,16)$. It was also demonstrated that $\mathrm{H}_{2} \mathrm{O}_{2}$ formation caused by bovine serum spermine oxidase (BSAO) (enzyme able to deaminate endogenous polyamines generating $\mathrm{H}_{2} \mathrm{O}_{2}$ and aldehyde) delivers deleterious effects in several human cancer cellular models, noticeable greater in multidrug resistance (MDR) tumor cells than in their wild-type counterpart (17-19). Since a variety of tumor cells, including MDR cells (P-glycoprotein expressing phenotype) produce very high amounts of ROS $(13,20)$ and in vivo many tumors appear resistant to oxidative stress and apoptosis, we dissected the influence of chronic sub-lethal DNA damage and DNA repair by $\mathrm{mSMOX}$ ectopically overexpressed in proficient Chinese hamster AA8 cell line and both deficient base-excision-repair (BER) EM9 (21) and transcriptioncoupled nucleotide-excision-repair (NER) UV61 (22) cell lines to represent cellular models of priming damage dose of ROS. Low doses of X-irradiation delivers challenging dose of damage evaluated at 6 and $24 \mathrm{~h}$ after exposure. Summarizing, the priming dose of ROS over-exposure by mSMOX provokes an adaptive response in N18TG2, AA8 and EM9 cell lines at $24 \mathrm{~h}$. In the UV61 cell line ROS, mSMOX did not deliver an adaptive response to radiation. According to Pelicano et al (23) the escalated ROS generation in cancer cells serves as an endogenous source of DNA-damaging agents, which promote genetic instability and development of drug resistance. Therefore, on the basis of an increased ROS production, from SPM enzymatic catabolism, in cancer cells therapeutic strategies have been suggested, which rely on the assumption that cancer cells, mainly MDR cells, are more sensitive to additional exposure to radicals than WT cells $(12,23)$. Once again, the pivotal roles played by SMOX and PA catabolism seem to evoke the biological processes of stress response, wherein balance is mandatory to live or to die (3). Thus, these alterations could represent a multitasking anticancer strategy, addressed not only to PA metabolism, but involving also radiation biology.

\section{Materials and methods}

Cell culture, radiation exposure and reagents. Reagents were from Sigma-Aldrich (Sigma-Aldrich, St. Louis, MO, USA), unless otherwise specified. Plastic-wares were from Nunc
(Nunc A/S, Roskilde, Denmark). A human colon adenocarcinoma cell line (LoVo WT), isolated from a metastatic nodule and its doxorubicin (DOX)-resistant variant (LoVo DX) were used in this study. Both cell lines were grown in monolayer in Ham's F12 medium (Gibco BRL, Life Technologies, Paisley, UK) supplemented with $10 \%$ fetal bovine serum (FBS) (Hyclone, Cramlington NE23, UK), 1\% L-glutamine (Gibco BRL, Life Technologies), $1 \%$ penicillin $(50 \mathrm{U} / \mathrm{ml})-$ streptomycin $(50 \mu \mathrm{g} / \mathrm{ml})$ (Gibco BRL, Life Technologies), $1 \%$ vitamins (Gibco BRL, Life Technologies) in a humidified atmosphere of $5 \% \mathrm{CO}_{2}$ in a water-jacketed incubator at $37^{\circ} \mathrm{C}$. The pleiotropic drug-resistant cell line LoVo DX was obtained from its drug-sensitive parental LoVo cell line, by exposure to increasing concentrations of DOX (Adriblastina, Pharmacia and Upjohn, Milan, Italy) (24). Transfection and growth conditions of pcDNA3 transfected (NP), pcDNA3SMOX transfected (NS) and mouse NB cell lines have been described $(15,16)$. AA8, EM9 and UV61 cell lines $(21,22)$ were a generous gift of Professor F. Palitti (Universita' della Tuscia, Italy). Stable transfection with pcDNA3 and pcDNA3SMOX were performed with Effectene (Qiagen) as described (16). Geneticin (G418) (300 mM) was the selection agent to isolate stable transfected pool of cells. X-irradiation of 1 and 10 cGy, were delivered by a Gilardoni CHF 320 G Unit (Gilardoni S.p.A., Mandello L., Italy) tested and complying with EU standards. Dose/rate was $0.1 \mathrm{~Gy} / \mathrm{min}$ at $250 \mathrm{KeV}$, 1,5 A, with $0.5 \mathrm{~mm} \mathrm{Cu}$ filter. Cells were irradiated on ice and fresh medium was replaced after exposure. Control cells were treated similarly, without irradiation. All experimental points were taken 6 and $24 \mathrm{~h}$ after irradiation.

NP, NS, AA8, EMO and UV61 proliferation assay (MTT). To analyse cell proliferation we followed the instructions of the manufacturer of the Cell Proliferation Kit I (Roche). We performed triplicate experiments with increasing number of cell/plate $\left(10^{3}, 2 \times 10^{3}, 3 \times 10^{3}\right.$ cell/plate) normalised to $10^{3}$. Cells were seeded the day before the experiments and treated with the MTT solution for $2 \mathrm{~h}$ at growth conditions. Isopropanol $(0.1 \mathrm{ml})$ with $0.04 \mathrm{~N} \mathrm{HCl}$ was added to each well to quench the red phenol colour and the absorbance was measured on an ELISA plate reader with a test wavelength of $570 \mathrm{~nm}$ and a reference wavelength of $630 \mathrm{~nm}$.

Reactive oxidative species and flow cytometry. Level of intracellular hydrogen peroxide was determined by flow cytometry (FCM) analysis of fluorescence intensity of 2',7'-dichlorodihydroflurescein ( $\left.\mathrm{H}_{2} \mathrm{DCFDA}\right)$ (Invitrogen). Briefly, cells were treated with $\mathrm{H}_{2} \mathrm{DCFDA}$ for $30 \mathrm{~min}$. At least $2 \times 10^{4}$ cells were analyzed by a FACSCalibur flow cytometer (Becton-Dickinson, San Josè, CA, USA), with laser excitation set at $495 \mathrm{~nm}$ and a 525-nm emission filter to detect green fluorescence. The negative control was obtained omitting fluorescence probe-mix from the reaction and auto-fluorescence was estimated. To study DNA content, cells were treated with propidium iodide (PI). At least $10^{5}$ cells were analyzed by FACSCalibur previously calibrated by CaliBRITE 3 beads (Becton-Dickinson), with laser excitation set at $488 \mathrm{~nm}$ and a 630-nm emission filter to detect red fluorescence. Level of 3'-terminal deoxy-transferase (TdT) by TUNEL method was used to detect apoptosis, following the manufacturer instruc- 
tions (In Situ Cell Death Fluorescent kit, Roche Diagnostic S.p.A., Monza, Italy). Laser excitation was set at $488 \mathrm{~nm}$ and emission was at 550-nm for FITC. As an auto-fluorescence control, a sample treated with label solution but without TdT was carried out for each set of analyses. FCM histograms were analysed by the Windows Multiple Document Interface (WinMDI ver. 2.8, The Scripps Research Institute, La Jolla, CA, USA) dedicated software.

BSAO purification. BSAO was purified from bovine blood as previously described (25). The purified enzyme moved as a single band on SDS/PAGE and all samples employed had a minimum specific benzylamine oxidase activity of $0.35 \mathrm{IU} /$ $\mathrm{mg}$, with IU defined as $\mu$ moles of substrate oxidized per min, assayed spectrophotometrically at $25^{\circ} \mathrm{C}$ by monitoring the formation of benzaldehyde at $250 \mathrm{~nm}$ absorbance $(\varepsilon=$ $\left.12,500 \mathrm{M}^{-1} / \mathrm{cm}^{-1}\right)$. The protein concentration was measured spectroscopically and from the $280 \mathrm{~nm}$ absorbance, assuming an absorption coefficient of $1.74 \mathrm{l} \mathrm{g}^{-1} / \mathrm{cm}^{-1}$.

LoVo WT and LoVo DX survival experiments. Cell survival experiments were carried out using confluent cells that had been incubated for $24 \mathrm{~h}$ at $37^{\circ} \mathrm{C}$ with fresh culture medium. Cells were harvested with EDTA in phosphate buffer saline (PBS) and then by addition of trypsin solution in PBS, washed by centrifugation and resuspended in PBS supplemented with $1 \%$ bovine serum albumin (BSA) (Sigma). Freshly harvested LoVo WT and LoVo DX cells $\left(10^{5} / \mathrm{ml}\right)$ were incubated at $37^{\circ} \mathrm{C}$ for varying time intervals in the presence of the following reagents, used alone or in combination: BSAO $\left(1.03 \times 10^{-4} \mu\right.$ moles $/ \mathrm{ml}$ corresponding to $6.98 \times 10^{-3} \mathrm{U} /$ $\mathrm{ml})$, spermine $(0-6 \mu \mathrm{M})$, catalase $(240 \mathrm{U} / \mathrm{ml})$ from bovine liver (Sigma), ALDH (EC 1.2.1.5) from yeast $(0.4 \mathrm{U} / \mathrm{ml})$ and nicotine adenin dinucleotide $\left(\mathrm{NAD}^{+}\right)(1.8 \mu \mathrm{g} / \mathrm{ml}$; BoehringerMannheim, Mannheim, Germany). Spermine (Fluka, Buchs, Switzerland) was freshly prepared before each experiment and, if used, added last. Cells were then centrifuged, washed in PBS-BSA and finally resuspended in $1 \mathrm{ml}$ PBS-BSA. The cells were then plated in tissue culture-coated Petri dishes $(60 \times 15 \mathrm{~mm})$ and incubated at $37^{\circ} \mathrm{C}$. Cytotoxicity was evaluated using a colony survival assay, thus determining the ability of cells to reproduce and form macroscopic colonies ( $>50$ cells). After three weeks, colonies were fixed with $96 \%$ ethanol, stained with methylene blue and counted manually. Percentage cell survival was determined as the ratio between the mean number of colonies in treated and control samples.

cDNA synthesis and real-time PCR. Total RNA was extracted from the hippocampus and frontal cortex with TRizol reagent (Invitrogen) and subjected to DNaseI treatment (Promega) according to the manufacturer's instructions. Two micrograms of total RNA were then used for cDNA synthesis, using SuperScript II (BRL Life Technologies) and random hexamer primers according to the manufacturer's instructions. One microliter of cDNA was used for amplification using the following primers: mGlu2 receptor: Bcl-2 forward, 5'-CTA CAGTGATGTCTCCATCC-3', reverse, 5'-AAAGCCTCAATG CCTGTCTC-3'; mGlu3 receptor: forward, 5'-CAAGTGAC TACAGAGTGCAG-3',reverse,5'-CTGTCACCAATGCTCAG CTC-3'; $\beta$-actin: BAX forward, 5'-TGAACCCTAAGGCCAA
CCGTG-3' reverse, 5'-GCTCATAGCTCTTCTCCAGGG-3'. Real-time quantitative PCR was performed using a $2 \mathrm{X}$ Supermix mixture (Bio-Rad) containing the double-stranded DNA Binding fluorescent probe SYBR Green and all necessary components except primers. Quantitative PCR conditions included an initial denaturation step of $94^{\circ} \mathrm{C} / 10 \mathrm{~min}$, followed by 40 cycles of $94^{\circ} \mathrm{C} / 15 \mathrm{sec}$ and $55^{\circ} \mathrm{C} / 15 \mathrm{sec}$. Standards, samples and negative controls (no template) were analyzed in triplicate. Concentrations of mRNA were calculated from serially diluted standard curves simultaneously amplified with the unknown samples and corrected for $\beta$-actin mRNA levels.

Statistical analysis. Significant difference at $\mathrm{p}<0.05$ was evaluated by the one-way ANOVA, followed by the multiple comparison Tukey post-hoc test (SPSS-11 statistical dedicated software - SPSS Inc., Chicago, IL, USA). Whisker box-plot graphs were obtained by open source software Gnumeric (Linux environment). All experiments were repeated three times unless otherwise indicated.

\section{Results}

mSMOX radiation and ROS in a neuroblastoma cell line. In previous studies, mSMOX activity was described to induce chronic sub-lethal DNA damage, with a 3-fold increase in oxo $^{8} \mathrm{dG}$ residues, but no increase in cell mortality. Upon 2- and 4-Gy doses of X-irradiation, SMOX transfected cells were sensitized and more prone to die than mock transfected cells. Treatments with increasing doses of MDL abolished such radiosensitive predisposition $(15,16)$. However, the level of X-ray delivered has to be considered as high doses, thus belonging to the LNT (linear no-threshold) theory of a linear dose-response. In the present study, we delivered a low dose of X-rays (10 cGy) (Fig. 1A, bar-graphs) with the relative cell cycle compartment composition, as determined by FCM. To confirm previous data, mSMOX alone does not apparently alter cell cycle and/or cell mortality, even in the presence of low dose irradiation at 6 and $24 \mathrm{~h}$ after exposure. Interestingly, when proliferation rates were detected by the more sensitive MTT assays at $6 \mathrm{~h}, \mathrm{mSMOX}$ associated with a hypersensitive dose-effect curve at low dose and with an adaptive response at higher dose (Fig. 1B).

mSMOX and radiation in AA8 parental, BER deficient EM9 and NER deficient UV61 cell lines. To dissect the influence of chronic sub-lethal DNA damage and DNA repair mechanisms, mSMOX was ectopically overexpressed in proficient Chinese hamster AA8 cell line and both deficient base-excision-repair EM9 cell line and deficient transcription-coupled nucleotideexcision-repair UV61 cell line to represent cellular models of priming damage dose of ROS. In Fig. 2A, proliferation MTT assays are represented as dose-effect curves related to low dose irradiation at 1 and $10 \mathrm{cGy}$, both determined at 6 and $24 \mathrm{~h}$ after exposure. In the AA8 and BER deficient EM9 cell lines, a hypersensitive reaction at 1-cGy exposure is detected at $6 \mathrm{~h}$, independently from SMOX overexpression. At $24 \mathrm{~h}$, the mock transfected AA8 cells followed a linear dose-effect curve. Contrarily, mSMOX transfected cells were resistant to both 1 and 10 cGy doses, showing a clear resistance to low dose radiation. In this case, $\mathrm{mSMOX}$ elicits an adaptive response 

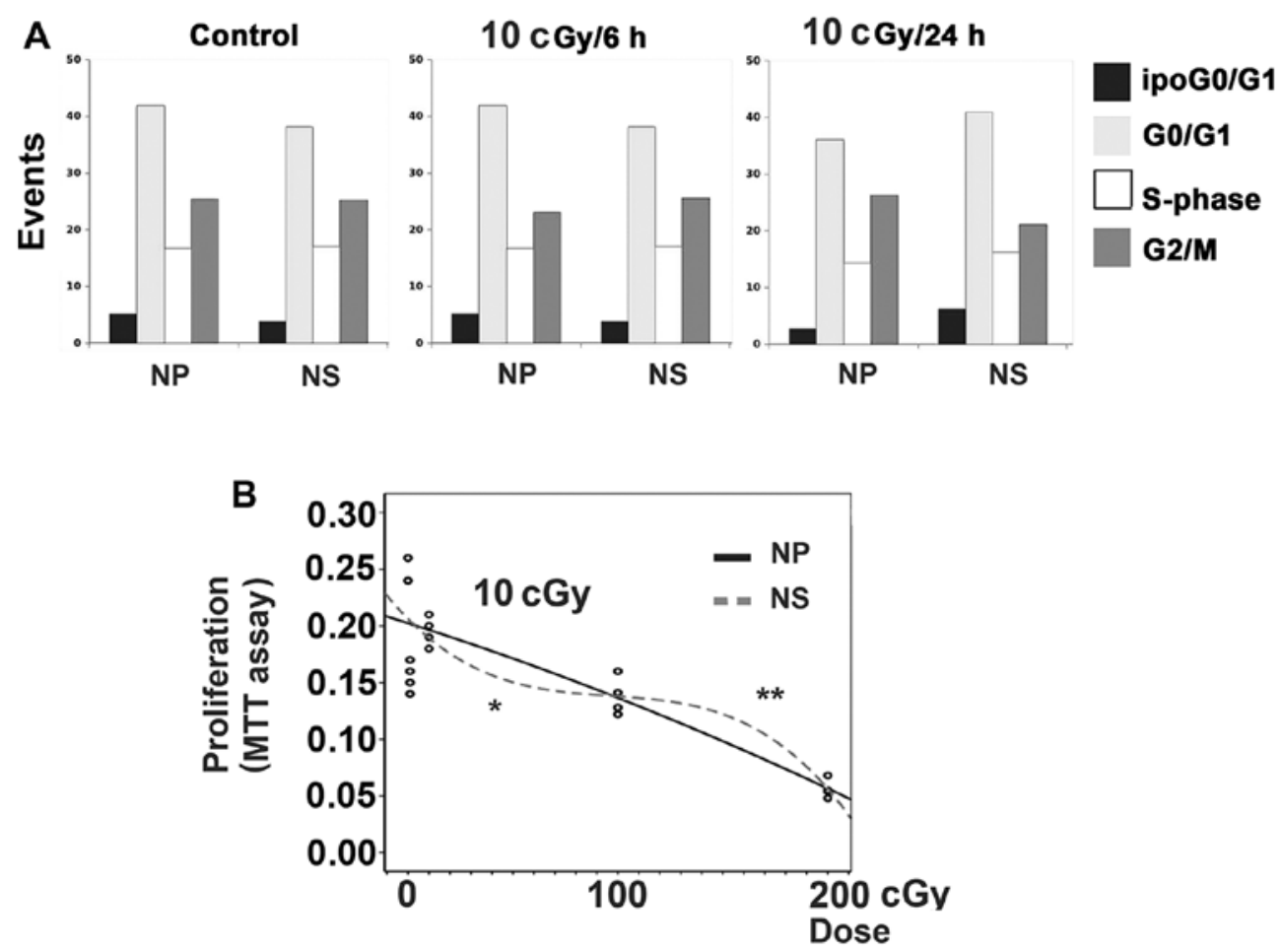

Figure 1. SMOX overexpression and low dose IR exposure in NB cell line. (A) Bar-graphs of relative cell cycle compartments determined by DNA content FCM. IR was delivered at $10 \mathrm{cGy}$ and analysis was performed at 6 and $24 \mathrm{~h}$ after exposure. (B) MTT proliferation assay performed at $6 \mathrm{~h}$ after 10,100 and 200 cGy (three replicas). NP, N18TG2 cells transfected with pcDNA3 plasmid (black); NS, N18TG2 cells transfected with pcDNA3/mSMOX plasmid (gray). "Hypersensitivity; ${ }^{* *}$ adaptive response.

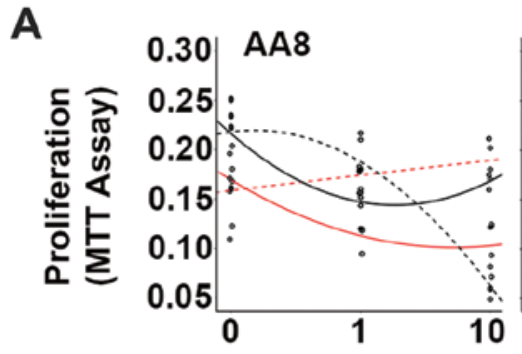

B

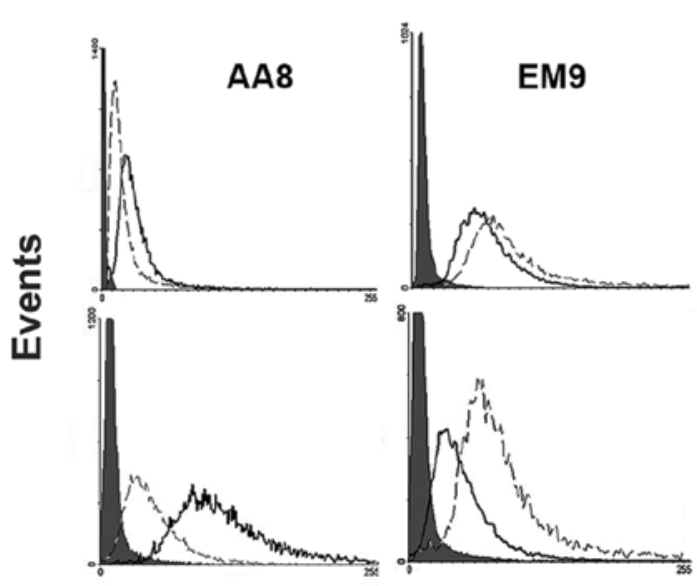

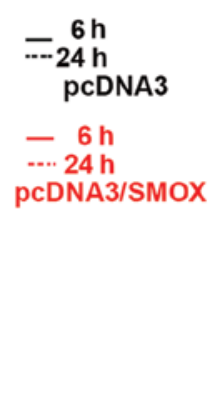

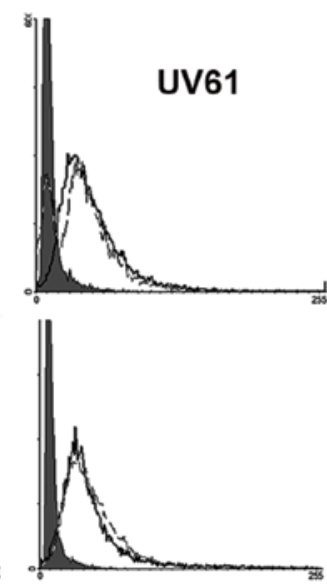

Control

$24 \mathrm{~h}$

Figure 2. SMOX overexpression in AA8, EM9, UV61 cell lines and low dose IR exposure. (A) MTT proliferation assay performed at 6 and 24 h after 1- and 10-cGy exposure (three replicas) in AA8, EM9, UV61 cell lines, as indicated. Black line, cell line transfected with pcDNA3 plasmid at 6 h; black dotted line, cell line transfected with pcDNA3 plasmid at $24 \mathrm{~h}$; gray line, corresponding cell line transfected with pcDNA3/mSMOX plasmid at 6 h; dotted gray line, corresponding cell line transfected with pcDNA3 plasmid at $24 \mathrm{~h}$. (B) Representative FCM histograms of NP (black) and NS (dashed) cell lines to analyse TUNEL reaction, at 0 and $10 \mathrm{cGy}$ exposure at $24 \mathrm{~h}$. Filled gray histograms represent autofluoresence. 


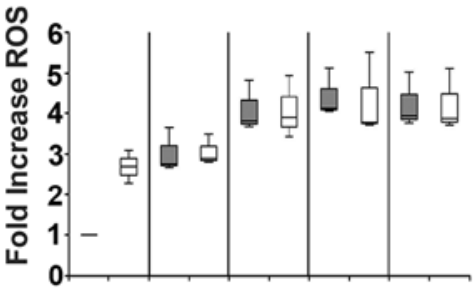

\begin{tabular}{|c|c|c|c|c|c|} 
Dose-cGy & - & 1 & 1 & 10 & 10 \\
\cline { 2 - 6 } Time-hours & - & 6 & 24 & 6 & 24 \\
\cline { 2 - 5 } & &
\end{tabular}
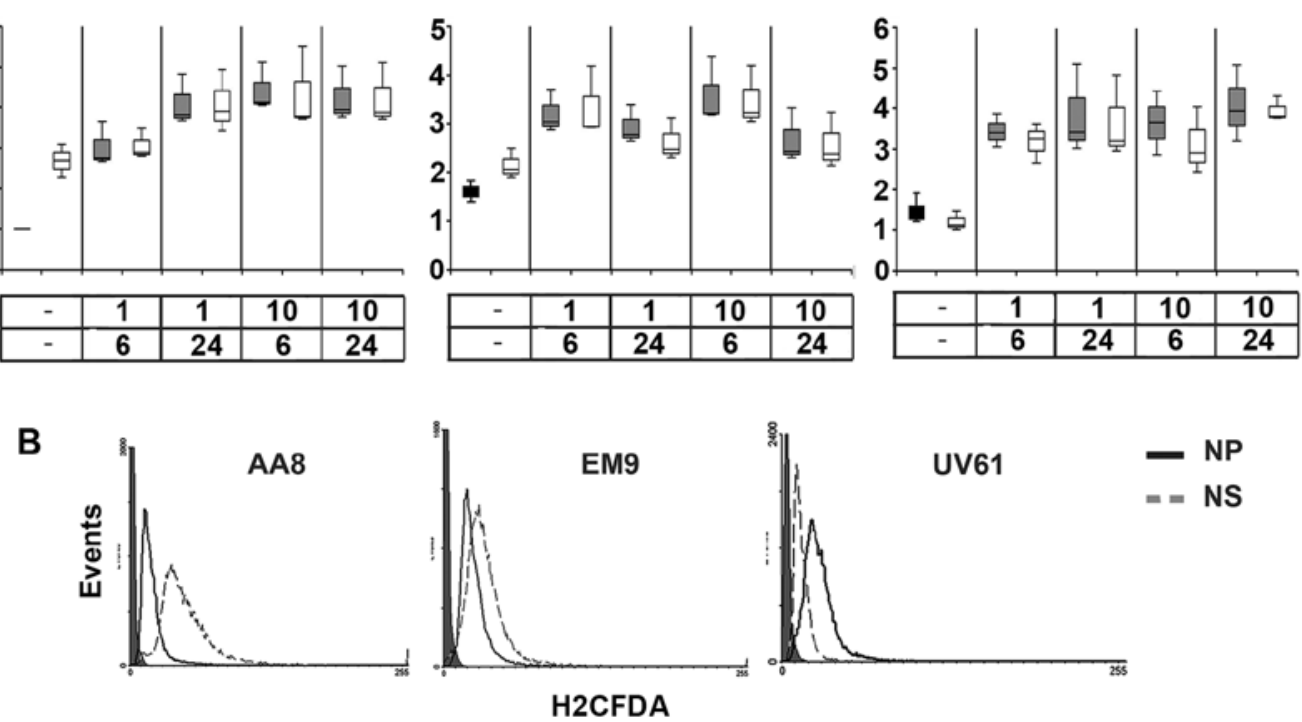

Figure 3. SMOX overexpression in AA8, EM9, UV61 cell lines and ROS. (A) Whisker box-plot representation of ROS fold increase. Dark gray, cell line transfected with pcDNA3 plasmid; white, cell line transfected with pcDNA3/mSMOX plasmid. " $p<0.05$. Bars represent $95 \%$ CI. (B) Representative ROS content by means of H2CFDA determined by FCM of NP (black) and NS (dashed) cell lines at control conditions. Filled gray histograms represent autofluoresence.

rendering cells more reactive against DNA damage. In the EM9 cells, mSMOX does not alter the dose-effect curves of cellular response to irradiation at $24 \mathrm{~h}$, being both more resistant than AA8 cells. However, the mSMOX overexpression provokes an additive damage with the BER deficiency upon low dose irradiation. In the UV61 cells, the $6 \mathrm{~h}$ hypersensitivity is registered only for mock transfected cells. At $24 \mathrm{~h}$, cells keep a consistent proliferation rate independently of mSMOX and irradiation doses. The overexpression of mSMOX seems to deliver an earlier adaptive response at $6 \mathrm{~h}$, although the proliferation-rates of UV61 cell line is much lower than AA8 and EM9 cell lines. According to the proliferation rates, we determined the course of apoptosis by means of TUNEL reaction. In Fig. 2B, representative FCM histograms are shown for the unirradiated and $24 \mathrm{~h}$ after $10-\mathrm{cGy}$ exposure. In the AA8 cell line, the adaptive response delivered by $\mathrm{mSMOX}$ is clearly evidenced by the different reduced amounts of apoptosis at 24 h. In the EM9 cells, SMOX provokes more damage and, consistently, TUNEL reaction is more evident for mSMOX transfected cells. In the UV61 cells, TUNEL was barely detectable with no influence by $\mathrm{mSMOX}$, probably also due to the low proliferation rates.

mSMOX and ROS in AA8 parental, BER deficient EM9 and NER deficient UV61 cell lines. The interconversion metabolism of SPM by SMOX produces hydrogen-peroxide and substantial evidence has addressed this fundamental aspect of SMOX activity to cause intracellular ROS (14-16). In Fig. 3A the fold increase of ROS induced by the mSMOX overexpression is represented as box-plot graph for all cell lines (representative histograms are shown in Fig. 3B). The augmented levels of ROS were significantly higher in AA8 and EM9 cell lines when transfected with mSMOX. The ROS level in the UV61 cell line is not influenced by mSMOX. More likely, UV61 are very sensitive to ROS overproduction, being deficient in NER

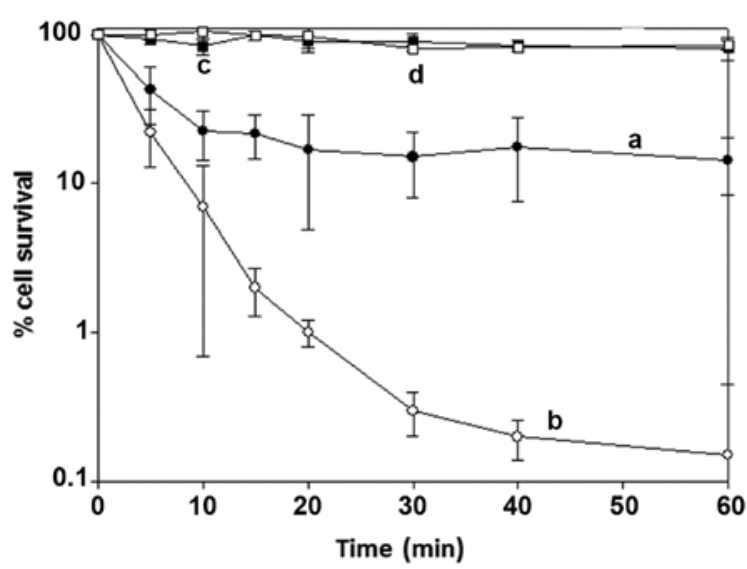

Figure 4. Effect of catalase on cytotoxicity induced by BSAO in the presence of spermine. LoVo WT (dark symbol, curve a) and LoVo DX (white symbol, curve b) cells were incubated at $37^{\circ} \mathrm{C}$ with purified BSAO $\left(6.98 \times 10^{-3} \mathrm{IU} / \mathrm{ml}\right)$ and exogenous spermine $(12 \mu \mathrm{M})$ and with catalase $(240 \mathrm{U} / \mathrm{ml})$ (dark WT and white DX symbols, curves c and d). Each point represents the mean value of the results of 2-5 plates of three or four experiments. The error bars indicate $\pm \mathrm{SD}$. When not shown, SD was smaller than the symbol.

repair system and any subtle ROS variation is not compatible with cell life. Radiation provokes an almost three times ROS increase in all cell lines tested, reaching a hypothetical threshold, which may represent a sort of life-death barrier.

LoVo WT and LoVo DX cell viability. Fig. 4 shows the percentage of cell survival versus the time of exposure to purified BSAO $\left(6.98 \times 10^{-3} \mathrm{U} / \mathrm{ml}\right)$ in the presence of exogenous spermine $(12 \mu \mathrm{M})$, with and without catalase, at $37^{\circ} \mathrm{C}$. In the presence of BSAO and spermine alone higher cytotoxicity was observed in LoVo DX than in LoVo WT cells. The percent cell survival decreased in both cell lines with increasing 
A

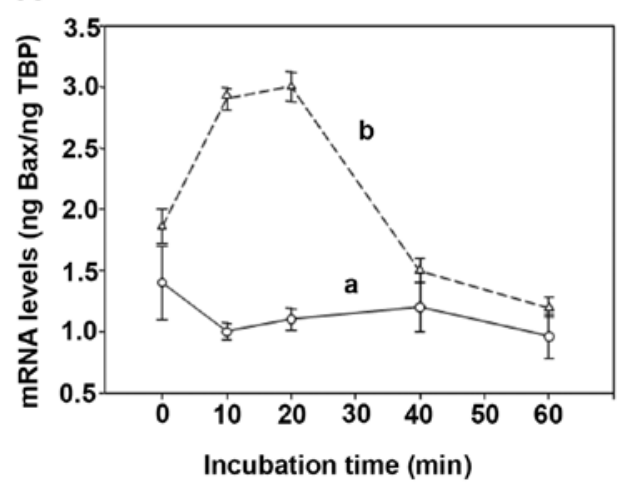

$B$

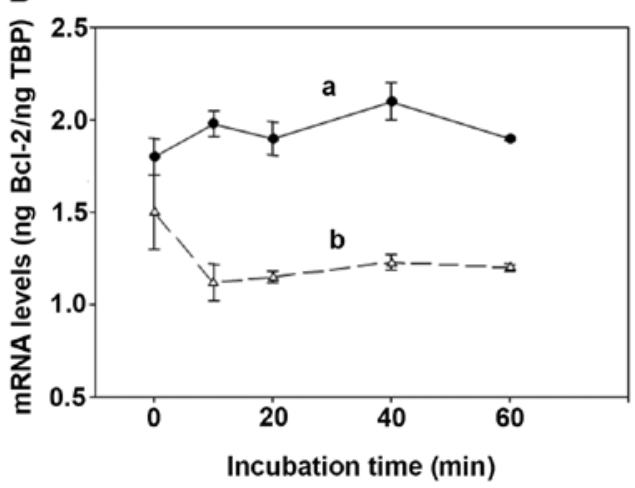

Figure 5. Bax and Bcl-2 mRNA levels expression after $\mathrm{H}_{2} \mathrm{O}_{2}$ administration. LoVo wild-type (curve a, dark symbol) and LoVo multidrug-resistant DX (curve b, white symbol) after treatment with $12 \mu \mathrm{M} \mathrm{H}_{2} \mathrm{O}_{2}$ for $60 \mathrm{~min}$. Standards, samples and negative controls (no template) were analyzed in triplicate. The error bars indicate $\pm \mathrm{SD}$. When not shown, SD was smaller than the symbol. (A) Bax expression. (B) Bcl-2 expression.

A

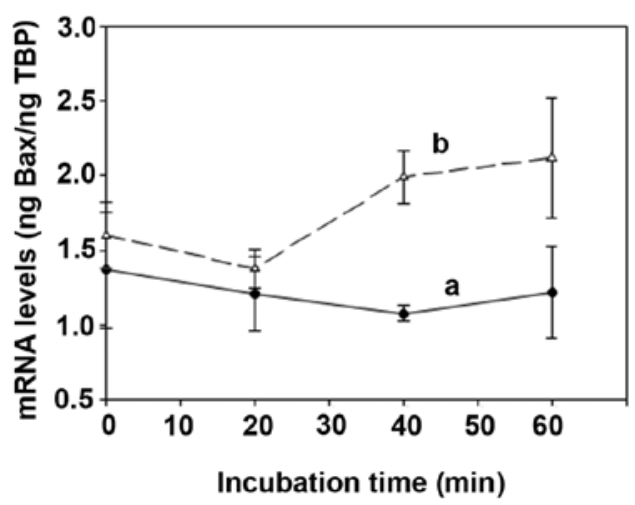

B

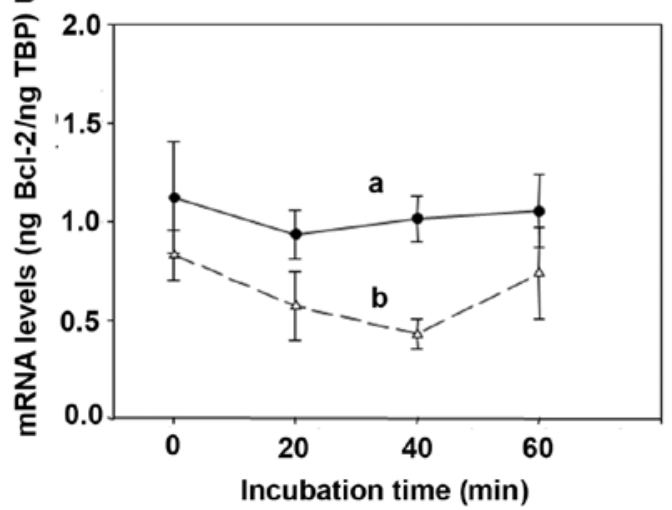

Figure 6. Bax and Bcl-2 mRNA level expression after acrolein administration. LoVo wild-type (curve a, dark symbol) and LoVo multidrug-resistant DX (curve b, white symbol) after treatment with $12 \mu \mathrm{M}$ acrolein for $60 \mathrm{~min}$. Standards, samples and negative controls (no template) were analyzed in triplicate. The error bars indicate \pm SD. When not shown, SD was smaller than the symbol. (A) Bax expression. (B) Bcl-2 expression.

exposure time, resulting in $\sim 18 \%$ in LoVo WT (Fig. 4, curve a) and $\sim 0.3 \%$ in LoVo DX cells (Fig. 4, curve b), after $60 \mathrm{~min}$ of incubation. In order to evaluate the contribution of each enzymatic oxidation product in the inhibition of cell growth, the experiments were performed in the presence of exogenous catalase, an enzyme which decomposes $\mathrm{H}_{2} \mathrm{O}_{2}$, or catalase and aldehyde dehydrogenase (ALDH) added simultaneously to the incubation mixture (data not shown). Catalase (240 U/ $\mathrm{ml}$ ) afforded a marked reduction of the cytotoxic effect, corresponding to $\sim 80 \%$ cell survival, on LoVo WT and LoVo DX cells (Fig. 4, curves c and d, respectively), probably due to the clearance of hydrogen peroxide, formed in the catalytic reaction by the enzyme. The result showed that $\mathrm{H}_{2} \mathrm{O}_{2}$ was not the sole toxic factor and that other products of the enzymatic oxidative deamination were involved, such as aldehyde(s), including acrolein spontaneously formed from the aminoaldehydes (27), an aspect still debated. The addition of exogenous NAD-dependent ALDH $(0.4 \mathrm{U} / \mathrm{ml})$ metabolized the aldehyde form to the corresponding carboxilic acid and prevented the toxic effects of the aldehyde(s) or acrolein. In fact, after addition of both exogenous enzymes, catalase and NAD-dependent ALDH, cytotoxicity was completely inhibited throughout the 60 min of incubation (data not shown).
Determination of the overexpression of the pro-apoptotic $B A X$ and of the pro-survival Bcl-2 genes induced by the spermine enzymatic oxidation products. Cytotoxicity induced on human cancer cells by bovine serum amine oxidase (BSAO) and spermine is mainly attributed to $\mathrm{H}_{2} \mathrm{O}_{2}$ generated by the enzymatic reaction. Results obtained on colon adenocarcinoma LoVo WT and LoVo DX cells by real-time PCR experiments, showed an increase in mRNA levels for the BAX pro-apoptotic gene in MDR cells after treatment of both LoVo cell lines with $12 \mu \mathrm{M}$ concentration of exogenous $\mathrm{H}_{2} \mathrm{O}_{2}$ (Fig. 5A). Whereas, LoVo cells treated with $12 \mu \mathrm{M}$ concentration of exogenous acrolein, showed only a slight overexpression of BAX at the last stage of the reaction in MDR cells (Fig. 6A). In these experimental conditions, the pro-survival Bcl-2 gene did not reveal any variation after LoVo WT and MDR cell treatment with either exogenous $\mathrm{H}_{2} \mathrm{O}_{2}$ (Fig. 5B) or acrolein (Fig. 6B). Therefore, the enhancement in mRNA levels for the BAX pro-apoptotic gene due to $\mathrm{H}_{2} \mathrm{O}_{2}$ and acrolein was more marked in LoVo MDR cells than in LoVo WT ones, in agreement with the clonogenic assay obtained by the treatment of LoVo cells with BSAO in presence of $12 \mu \mathrm{M}$ spermine concentration, as previously demonstrated by the authors. 


\section{Discussion}

In this study, we used the chronic sub-lethal damage driven by ectopical overexpression of mSMOX as priming dose of cellular stress, followed by two challenging low doses of X-irradiation, to evaluate the mSMOX capability to elicit an adaptive or additive response in cellular models. Moreover, we studied the influence of the in situ formation of cytotoxic ROS formed by BSAO and spermine to differentiate the MDR response in contrast with wild-type human tumor cell lines. Ionising radiation induces clustered DNA damage, defined as two or more lesions within one or two helical turns of the DNA double-helix by a single radiation track $(28,29)$. The primary cellular repair mechanism is the base excision repair (BER) pathway to repair base lesions, AP sites and SSBs which are induced in cells either endogenously or by IR $(30,31)$. In our models, the overexpression of mSMOX as priming dose of cellular stress is mainly ROS and DNA suffers oxidative damage from free radicals produced in living cells. This damage, if not correctly repaired, can lead to genomic instability and increased risk of developing cancer. To dissect if chronic sub-lethal DNA damage driven by SMOX overexpression could deliver adaptive or additive response, we force SMOX expression in different DNA repair deficient cellular models, such as EM9 (BER-deficient) and UV61 (NER-deficient) cell lines. The challenging doses of $\mathrm{X}$-irradiation were in the low dose range to evidence the influence of SMOX activity, mainly related to proliferation and survival. In fact, doses $<10$ cGy do not deliver any mutation and genomic instability (32), thus are out of the scope of this study. In NB cell line, SMOX delivered an effect not in agreement with the LNT theory, causing hypersensitivity at lower dose and adaptive response at the higher dose of $10 \mathrm{cGy}$. In the AA8 and BER deficient EM9 cell lines, mSMOX elicits an adaptive response rendering cells less sensitive to DNA damage. Moreover, at the lower dose of $1 \mathrm{cGy}, \mathrm{SMOX}$ is additive to BER deficiency, in agreement with that expected, since BER is the principal mechanism of DNA repair both for IR and ROS mediated DNA damage. In the UV61 cells, the overexpression of mSMOX seems to deliver an earlier adaptive response at $6 \mathrm{~h}$. NER deficient cell line seems take advantage to the priming damage of SMOX to rescue IR damage, as NER is not primarily involved in this kind of DNA repair. Controversially, tumor cells acquire resistance to grow in an oxidative environment, developing also a MDR mechanism to overwhelm chemotherapy. However, in recent years, we observed that cytotoxicity induced by ROS, downstream the spermine metabolites by BSAO, was greater in human tumor MDR LoVo cells than the wild-type counterpart, Agostinelli et al $(18,33)$. In addition, our findings also showed that ROS formed by the combination $\mathrm{BSAO} /$ spermine are not only able to prevent tumor cell growth, but also prevents mass tumor growth. MDR mitochondrial damage observed by mitochondrial membrane depolarization and transmission electron microscopy (TEM) was attributed to the cytotoxic effects induced by ROS, generated during the treatment, aldehyde(s) also contributed to cytotoxicity, but at a later stage of the reaction and to a lesser extent $(\sim 20 \%)$, as demonstrated in the presence of aldehyde dehydrogenase
$(12,13,34)$. In the present study, we further confirmed these results by real-time PCR experiments. Increasing mRNA levels for the BAX pro-apoptotic gene mainly was observed due to $\mathrm{H}_{2} \mathrm{O}_{2}$ and was again more evident in LoVo MDR cells than in their WT counterparts. In conclusion, SMOX could deliver a therapeutic gain when forced in NB, parental and cancer cells with impaired BER repair mechanism at low, fractionated dose of IR. Contrarily, in cells with deficiency in NER repair mechanisms, SMOX could play an adaptive role to overwhelm DNA damage by IR and be deleterious for therapy. In treatment with chemotherapy alone, cytotoxic PA metabolites might be important as a new approach in antineoplastic therapy in combating cancer, particularly against MDR cancer cells and this represents an aspect of particular importance with regard to the potential therapeutic applications of ROS, since conventional cancer therapy suffers from the development of drug resistance (35).

\section{Acknowledgements}

This study was funded in part by the Italian MIUR (Ministero dell'Istruzione, dell'Università e della Ricerca), by Istituto Superiore di Sanità 'Project Italy-USA', by Istituto Pasteur Fondazione Cenci Bolognetti. The authors are indebted with Professor F. Palitti (Univ. Tuscia, Viterbo, Italy) for supplying NER and BER deficient cell lines.

\section{References}

1. Ames BN, Shigenaga MK and Hagen TM: Oxidants, antioxidants and the degenerative diseases of aging. Proc Natl Acad Sci USA 90: 7915-7922, 1993.

2. Chance B, Sies H and Boveris A: Hydroperoxide metabolism in mammalian organs. Physiol Rev 59: 527-605, 1979.

3. Schumacker PT: Reactive oxygen species in cancer cells: live by the sword, die by the sword. Cancer Cell 10: 175-176, 2006.

4. Ghavami S, Eshragi M Ande SR, et al: S100A8/A9 induces autophagy and apoptosis via ROS-mediated cross-talk between mitochondria and lysosomes that involves BNIP3. Cell Res 20: 314-331, 2010.

5. Wong CH, Iskandar KB, Yadav SK, Hirpara JL, Loh T and Pervaiz S: Simultaneous induction of non-canonical autophagy and apoptosis in cancer cells by ROS-dependent ERK and JNK activation. PLoS One 5: e9996, 2010.

6. Kamata H, Honda S, Maeda S, et al: Reactive oxygen species promote TNFalpha-induced death and sustained JNK activation by inhibiting MAP kinase phosphatases. Cell 120: 649-661, 2005.

7. Wallace HM, Fraser AV and Hughes A: A perspective of polyamine metabolism. Biochem J 376: 1-14, 2003.

8. Casero RA Jr and Marton LJ: Targeting polyamine metabolism and function in cancer and other hyperproliferative diseases. Nat Rev Drug Discov 6: 373-390, 2007.

9. Ploszaj T, Motyl T, Zimowska W, Skierski J and Zwierzchowski L: Inhibition of ornithine decarboxylase by $\alpha$-difluoromethylornithine induces apoptosis in $\mathrm{HC} 11$ mouse mammary epithelial cells. Amino Acids 19: 483-496, 2000

10. Seiler N and Raul F: Polyamines and apoptosis. J Cell Mol Med 9: 623-642, 2005.

11. Amendola R, Cervelli M, Fratini E, Polticelli F, Sallustio DE and Mariottini P: Spermine metabolism and anticancer therapy. Cur Cancer Drug Targets 9: 118-130, 2009.

12. Calcabrini A, Arancia G, Marra M, et al: Enzymatic oxidation products of spermine induce greater cytotoxic effects on human multidrug-resistant colon carcinoma cells (LoVo) than on their wild-type counterparts. Int J Cancer 99: 43-52, 2002.

13. Arancia G, Calcabrini A, Marra M, et al: Mitochondrial alterations induced by serum amine oxidase and spermine on human multidrug resistant tumor cells. Amino Acids 26: 273-282, 2004. 
14. Pledgie A, Huang Y, Hacker A, et al: Spermine oxidase SMO(PAOh1), Not N1-acetylpolyamine oxidase PAO, is the primary source of cytotoxic $\mathrm{H}_{2} \mathrm{O}_{2}$ in polyamine analog-treated human breast cancer cell lines. J Biol Chem 280: 39843-39851, 2005.

15. Bianchi M, Bellini A, Cervelli M, et al: Chronic sub-lethal oxidative stress by spermine oxidase over activity induces continuous DNA repair and hypersensitivity to radiation exposure. Biochim Biophys Acta 1773: 774-783, 2007.

16. Amendola R, Bellini A, Cervelli M, et al: Direct oxidative DNA damage, apoptosis and radio sensitivity by spermine oxidase activities in mouse neuroblastoma cells. Biochim Biophys Acta Rev Cancer 1775: 15-24, 2005.

17. Agostinelli E, Arancia G, Dalla Vedova L, et al: The biological functions of polyamine oxidation products by amine oxidises: perspectives of clinical applications. Amino Acids 27: 347-358, 2004.

18. Agostinelli E, Dalla Vedova L, Belli F, Condello M, Arancia G and Seiler N: Sensitization of human colon adenocarcinoma cells (LoVo) to reactive oxygen species by lysosomotropic compounds. Int J Oncol 29: 947-955, 2006.

19. Agostinelli E, Condello M, Molinari A, Tempera G, Viceconte N and Arancia G: Cytotoxicity of spermine oxidation products to multidrug resistant melanoma cells (M14 ADR2): sensitisation by MDL 72527, a lysosomotropic compound. Int J Oncol 35: 485-498, 2009.

20. Soares FA, Shaugnessy SG, MacLarkey WR and Orr FW: Quantification and morphologic demonstration of reactive oxygen species produced by Walker 256 tumor cells in vitro and during metastasis in vivo. Lab Invest 71: 480-489, 1994.

21. Thompson LH, Brookman KW, Dillehay LE, et al: A CHO-cell strain having hypersensitivity to mutagens, a defect in DNA strand-break repair and an extraordinary baseline frequency of sister-chromatid exchange. Mutat Res 95: 427-440, 1982.

22. Thompson LH, Salazar EP, Brookman KW, et al: Recent progress with the DNA repair mutants of Chinese hamster ovary cells. J Cell Sci (Suppl 6): 97-110, 1987.

23. Pelicano H, Carney D and Huang P: ROS stress in cancer cells and therapeutic implication. Drug Resist Updat 7: 97-110, 2004
24. Grandi M, Geroni C and Giuliani FC: Isolation and characterization of a human colon adenocarcinoma cell line resistant to doxorubicin. Br J Cancer 54: 515-518, 1986

25. Turini P, Sabatini S, Befani O, et al: Purification of serum amine oxidase. Anal Biochem 125: 294-298, 1982.

26. Bahn S, Mimmack M, Ryan M, et al: Neuronal target genes of the neuron-restrictive silencer factor in neurospheres derived from fetuses with Down's syndrome: a gene expression study. Lancet 359: 310-315, 2002.

27. Sharmin S, Sakata K, Kashiwagi K, et al: Polyamine cytotoxicity in the presence of bovine serum amine oxidase. Biochem Biophys Res Commun 282: 228-235, 2001.

28. Regulus P, Duroux B, Bayle PA, et al: Oxidation of the sugar moiety of DNA by ionizing radiation or bleomycin could induce the formation of a cluster DNA lesion. Proc Natl Acad Sci USA 104: 14032-14037, 2007.

29. Eccles LJ, Lomax ME and O'Neill P: Hierarchy of lesion processing governs the repair, double-strand break formation and mutability of three-lesion clustered DNA damage. Nucleic Acids Res 38: 1123-1134, 2010.

30. Hitomi K, Iwai S. and Tainer JA: The intricate structural chemistry of base excision repair machinery: implications for DNA damage recognition, removal and repair. DNA Repair 6: 410-428, 2007.

31. Zharkov DO: Base excision DNA repair. Cell Mol Life Sci 65: 1544-1565, 2008

32. Maxwell CA, Fleisch MC, Sylvain V, et al: Targeted and nontargeted effects of ionizing radiation that impact genomic instability. Cancer Res 68: 8304-8831, 2008.

33. Agostinelli E, Belli F, Molinari A, et al: Toxicity of enzymatic oxidation products of spermine to human melanoma cells (M14): sensitization by heat and MDL 72527. Biochim Biophys Acta 1763: 1040-1050, 2006.

34. Agostinelli E and Seiler N: Lysosomotropic compounds and spermine enzymatic oxidation products in cancer therapy (Review). Int J Oncol 31: 473-484, 2007.

35. Agostinelli E and Seiler N: Non-irradiation-derived reactive oxygen species (ROS) and cancer. Therapeutic implications. Amino Acids 31: 341-355, 2006. 